\title{
Comparison of Continuous Adductor Canal and Femoral Nerve Blocks for Analgesia and Return of Quadriceps Function After Anterior Cruciate Ligament Reconstruction in Adolescent Patients
}

\author{
Erica L. Holland, M.D., Robin E. Robbins, M.D., \\ Daniel K. Low, B.M.B.S., M.R.C.P.C.H., F.R.C.A., \\ Adrian T. Bosenberg, M.B. Ch.B. FFA(SA), Viviana Bompadre, Ph.D., and \\ Gregory A. Schmale, M.D.
}

\begin{abstract}
Purpose: To compare early pain relief and late quadriceps function after anterior cruciate ligament reconstruction (ACLR) with hamstring autograft in adolescent patients treated with either a continuous femoral nerve block (cFNB) or continuous adductor canal block (CACB). Methods: We retrospectively reviewed a consecutive series of adolescent patients who underwent ACLR and received either a CACB or cFNB for postoperative pain management. Over a 1-year period, all patients underwent ACLR with cFNBs. Over the subsequent 9 months, all patients underwent their ACLR with cACBs. Patient demographics, postoperative pain scores, opioid consumption, satisfaction and complications, and dates and results of quadriceps function derived at the Return to Sports evaluation were compared. Results: Ninety-one patients $(53 \mathrm{cFNB}, 38 \mathrm{cACB}$ ) were reviewed. There were no differences in the demographics of the 2 groups. There were no statistically significant differences between groups in variations in postoperative pain scores $(P=.21)$, or satisfaction with the blocks $(P=.93)$. Patients in the cFNB group consumed a greater number of opioid doses on postoperative day 3 $(2.2 \pm 2.1$ doses $\mathrm{CFNB}, 1.1 \pm 1.6$ doses $\mathrm{CACB}, P=.03)$ and a greater number of opioid doses overall for postoperative days 1 to 3 (mean $6.8 \pm 5.3$ doses $c F N B, 3.8 \pm 2.1$ doses $\mathrm{CACB}, P=.03$ ). There was no difference in time to return of acceptable quadriceps strength and function when comparing the 2 groups $(30.9 \pm 7.7$ weeks cFNB, $28.9 \pm 6.6$ weeks cACB, $P=$ .087). Conclusions: We found few differences in postoperative analgesic requirements when comparing patients who underwent ACLR with hamstring autograft with a cACB to those who underwent a similar procedure with a cFNB. Return of quadriceps strength and function by six months did not appear to vary with regional technique, either cACB or cFNB, employed at surgery. Level of Evidence: III, Retrospective comparative study.
\end{abstract}

A nterior cruciate ligament reconstruction (ACLR) is a common procedure performed primarily in an ambulatory setting in which adequate postoperative

From the Department of Anesthesiology and Pain Medicine (E.L.H., D.K.L., A.T.B.) and Department of Orthopedics and Sports Medicine (V.B., G.A.S.), Seattle Children's, Seattle, Washington; and Department of Anesthesiology, Legacy Emanuel Medical Center, Portland, Oregon (R.E.R.), U.S.A.

The authors report the following potential conflicts of interest or sources of funding: D.K.L. reports other from MDmetrix, outside the submitted work. Full ICMJE author disclosure forms are available for this article online, as supplementary material.

Received September 24, 2019; accepted January 9, 2020.

Address correspondence to Gregory A. Schmale, M.D., Department of Orthopedics and Sports Medicine, M/S MB.10.620, P.O. Box 5371, Seattle, WA 98145-5005.E-mail: gschmale@uw.edu

(C) 2019 THE AUTHORS. Published by Elsevier Inc. on behalf of the Arthroscopy Association of North America. This is an open access article under the CC BY-NC-ND license (http://creativecommons.org/licenses/by-nc-nd/4.0/). 2666-061X/191133

https://doi.org/10.1016/j.asmr.2020.01.001 analgesia is an essential component of patient satisfaction, reliable discharge, and patient safety and functional rehabilitation. ${ }^{1-6}$ As immediate postoperative weight-bearing has been shown to decrease early postoperative pain, and postoperative pain levels are inversely associated with postoperative function, ${ }^{6}$ the advantages of motor-sparing peripheral nerve blocks enabling early weight-bearing become obvious. ${ }^{7}$ Early weight-bearing and elimination of immobilizing braces is commonly employed after ACLR. ${ }^{8}$ Although the use of peripheral nerve blocks for ACLR has increased significantly in recent years, consensus around the optimal pain management strategy after ACLR is lacking. ${ }^{1}$

A continuous femoral nerve block (cFNB) is one technique for postoperative analgesia after ACLR in pediatric patients. The advantages of continuous nerve blocks over single-shot blocks include decreasing 
"rebound pain" or the early discomfort associated with wearing off of the block within the first 24 hours. ${ }^{9,10}$ However, femoral nerve blocks may result in early and potentially prolonged quadriceps muscle weakness, ${ }^{11-13}$ leading to concerns about early mobilization and increased risk of postoperative falls. ${ }^{14,15}$ Prolonged weakness as measured by fast isokinetic knee extension strength at 6 months and increased ACL rupture rates after femoral nerve block in the first year after surgery also have been described. ${ }^{16}$

Continuous adductor canal blocks (cACBs) have emerged as an appealing alternative to CFNB, because they produce a predominantly sensory nerve block of the saphenous nerve. Although the femoral nerve blocks (FNBs) may affect all 4 quadriceps muscles, the only motor nerve traversing the adductor canal is the nerve to the vastus medialis muscle; thus, the cACB is able preserve quadriceps muscle strength compared with cFNB with similar analgesic efficacy. ${ }^{17-19}$ An adductor canal nerve block also would reduce the risk of injury to the motor branches of the nerves to the rectus femorus, vastus lateralis, and vastus intermedius, at risk with an FNB. Long-standing knee extensor weakness from FNBs has been reported and would be potentially debilitating for an athlete. ${ }^{20}$

Our purpose was to compare pain relief and quadriceps function (QF) after ACLR with hamstring autograft in adolescent patients treated with either a cFNB or cACB. We hypothesized that CACB compared with CFNB would result in decreased time to return of acceptable QF in tests of sports readiness and, hence, a greater proportion of patients with adequate QF by 6 months post-ACLR, without compromising early postoperative analgesia.

\section{Methods}

This was an institutional review board-approved retrospective review (Human Subjects approved by Seattle Children's: IRB STUDY00000999) of a consecutive series of adolescent patients who underwent ACLR between January 2016 and September 2018 at one institution by one surgeon and received either a CACB or CFNB for postoperative pain management. Before January 1, 2017, all ACLRs were performed with a cFNB, with a department-wide switch to $\mathrm{CACB}$ after this date. There were no changes in patient education or medications prescribed at discharge throughout the study period. Patient age, sex, American Society of Anesthesiologists physical status classification, primary and secondary procedures, procedure laterality, postoperative day (POD) 1, 2, and 3 opioid consumption, pain scores, overall satisfaction data and complications, and the date and results of the Return to Sports (RTS) evaluation were collected by manual chart review of the electronic medical record.
Our criteria for inclusion were a primary ACLR using a hamstring autograft, with or without meniscus repair or medial collateral ligament imbrication. Exclusion criteria included inadequate data in the medical record regarding postoperative pain (fewer than 2 of 3 postoperative days); reconstruction with a graft material other than hamstring autograft; a revision reconstruction, a multiligament reconstruction, a lateral extraarticular tenodesis procedure; previous ACLR on the contralateral knee; or other injuries or additional surgeries during the rehabilitation period that delayed rehabilitation (Fig 1). Surgical procedures were performed by a single fellowship-trained pediatric orthopaedic surgeon with a certificate of added qualification in Sports Medicine.

\section{Operative Technique}

ACLRs were performed arthroscopically, with grafts placed through a tibial bone tunnel and an independently drilled femoral tunnel. Suspensory fixation was employed on the femur, and a screw and washer provided post-fixation on the tibia. When performed, meniscus repairs were either all-inside, outside-in, or inside-out, the latter via a small posteromedial or posterolateral incision. Medial collateral ligament imbrications used the hamstring harvest incision for placement of imbricating sutures. Skeletally immature patients underwent transphyseal reconstructions with all-metaphyseal fixation. The postoperative protocol encouraged patients to weight-bear as tolerated with their brace locked in extension for the first week, after which the brace was opened by their physical therapist as flexion improved. A cryotherapy device was used for all patients for the first 4 days, and ad libitum to follow.

\section{Anesthetic Technique}

All patients received a general anesthetic with induction and maintenance medications at the discretion of the anesthesiologist. In addition to acetaminophen and ketorolac, short- or long-acting opioids were administered when intraoperative analgesia was determined to be inadequate, based on hemodynamic responses.

\section{Regional Technique}

Ultrasound-guided cFNB or CACB was placed after induction of anesthesia (as this is standard of practice in the pediatric population) and before incision by one of a team of pediatric fellowship-trained anesthesiologists, all of whom perform these blocks on a regular basis. At the discretion of the anesthesiologist, the initial bolus of local anesthetic consisted of $0.2 \%$ or $0.5 \%$ ropivacaine. During surgery, a continuous infusion of $0.2 \%$ ropivacaine was initiated at a weight-based rate (ranging from $4-6 \mathrm{~mL} / \mathrm{h}$ ), intended to last for 3 postoperative days, delivered by a disposable ON-Q pump (Avanos 


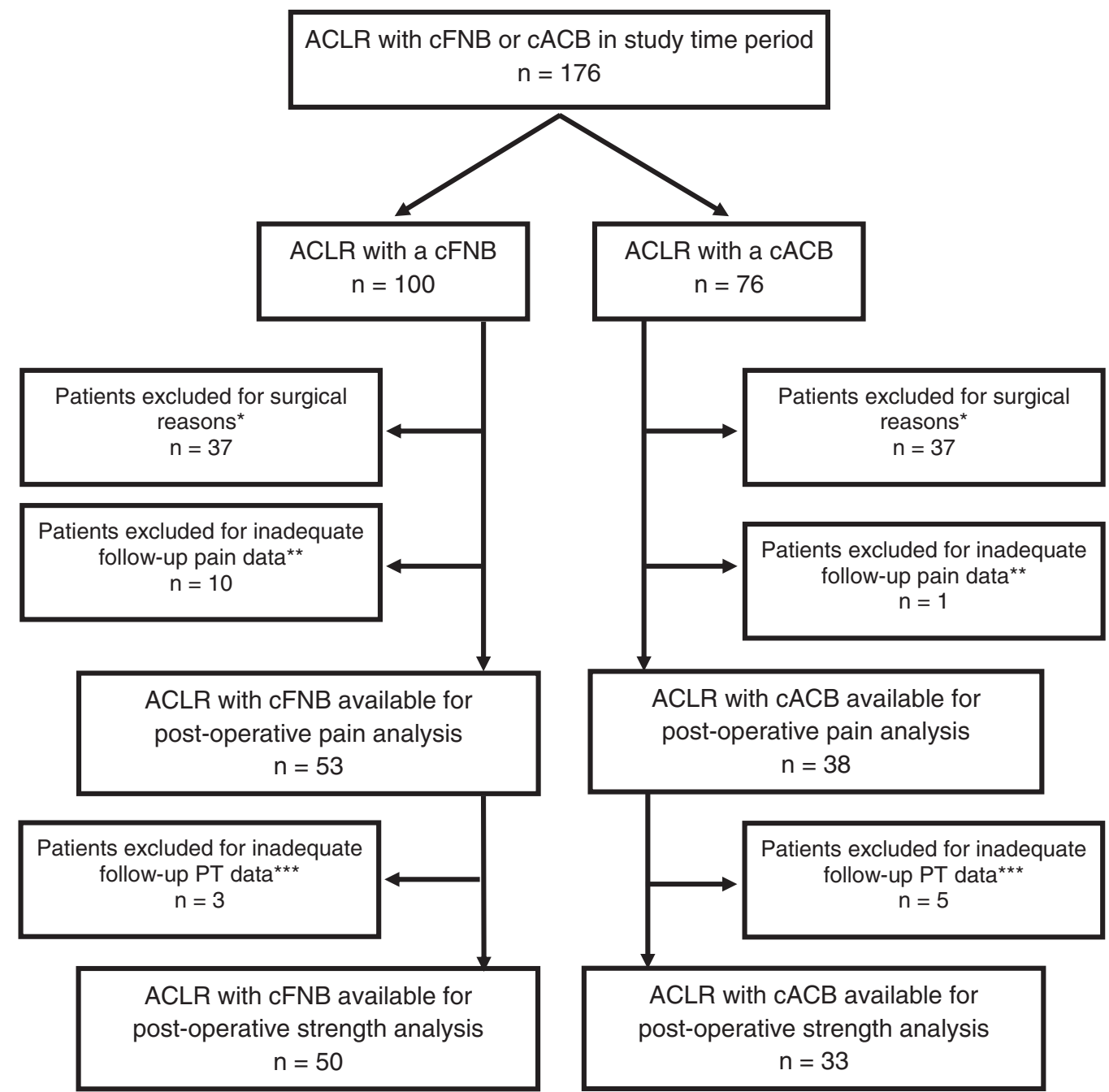

Fig 1. Exclusion flow diagram. *Surgical exclusion: Patients excluded due to revision nature of surgery, additional bony or ligamentous reconstruction surgery, non-hamstring ACL graft material, or previous contralateral ACLR. **Inadequate early follow-up: Patients excluded for inadequate postoperative pain data. ${ }^{* *}$ Inadequate late follow-up: Patients excluded for inadequate postoperative PT data. (ACL, anterior cruciate ligament; ACLR, anterior cruciate ligament reconstruction; cACB, continuous adductor canal block; cFNB, continuous femoral nerve block; PT, physical therapy.)

Medical Inc., Irvine, CA). All patients additionally received a subgluteal sciatic single shot block for posterior knee pain with either $0.2 \%$ or $0.5 \%$ ropivacaine.

Patients were discharged with prescriptions for acetaminophen, ibuprofen, and oxycodone for pain relief and were instructed to take scheduled acetaminophen and ibuprofen independent of pain level, with oxycodone used only on an as-needed basis. The dose of oxycodone prescribed was $5 \mathrm{mg}$ for patients less than $75 \mathrm{~kg}$ and $7.5 \mathrm{mg}$ for patients greater than $75 \mathrm{~kg}$.

\section{Postoperative Protocol}

Families were contacted by either an attending anesthesiologist or fellow on the Regional Anesthesia Service on POD 1, 2, and 3, or until it was confirmed the catheter was safely removed and full sensory/motor function had returned. Information collected included pain scores, opioid and other medication usage, catheter dressing integrity, signs of local anesthetic toxicity, and overall satisfaction. Subjective satisfaction data asked caregivers to choose 1 of 4 evaluations regarding the peripheral nerve catheter: very satisfied, somewhat satisfied, somewhat unsatisfied, and very unsatisfied.

Patients were prescribed weekly physical therapy, performed at either our institution or off-site. Patients returned for physical examinations at 7-14 days, 4-7 weeks, 3 months, and 6 months postoperatively, and annually thereafter. At approximately 6 months postoperatively, an RTS assessment was performed to establish whether the patient was safe to return to sports as well as to guide further rehabilitation. Our RTS readiness assessment consisted of objective evaluations of strength, coordination, agility, endurance, and balance as described by Barber-Westin and Noyes, ${ }^{21}$ 
Table 1. Patient and Surgical Characteristics

\begin{tabular}{|c|c|c|c|}
\hline & cFNB $(n=53)$ & $\mathrm{cACB}(\mathrm{n}=38)$ & $P$ Value \\
\hline Age at surgery, y & $15.0 \pm 1.9$ & $15.3 \pm 1.6$ & .332 \\
\hline Range, y & $11-21$ & 13-19 & \\
\hline Sex & & & .294 \\
\hline Female & $22(42 \%)$ & $20(53 \%)$ & \\
\hline \multicolumn{4}{|l|}{ ASA class } \\
\hline 1 & $37(70 \%)$ & $26(68 \%)$ & .887 \\
\hline Right & $30(57 \%)$ & $18(47 \%)$ & \\
\hline Left & $23(43 \%)$ & $20(53 \%)$ & \\
\hline \multicolumn{4}{|l|}{ Secondary procedure, $\mathrm{n}$} \\
\hline None (only primary ACLR) & $24(45 \%)$ & $19(50 \%)$ & .830 \\
\hline Meniscus repair (lateral, medial or both) & $20(38 \%)$ & $16(42 \%)$ & \\
\hline Medial collateral ligament imbrication \pm meniscus repair & $6(11 \%)$ & $3(8 \%)$ & \\
\hline
\end{tabular}

NOTE. Data are reported as mean \pm standard deviation or $\mathrm{n}(\%)$.

ACLR, anterior cruciate ligament reconstruction; ASA, American Society of Anesthesiology physical status classification; cACB, continuous adductor canal block; cFNB, continuous femoral nerve block.

*Lateral lengthening for patellar stabilization, additional incision for hamstring harvest, patellar tendon ossicle excision.

Thomee et al., ${ }^{22}$ and Hewett et al., ${ }^{23}$ performed at our Sports Physical Therapy center by a small cadre of trained physical therapists. Patients were tested on isokinetic strength of both limbs for hip abduction, knee extension, and knee flexion; underwent a Y-balance test; were examined in drop jumps for dynamic knee valgus; and underwent single and triple leg hops of both limbs, with a desired Limb Symmetry Index of $90 \%$ or better. With the objective of specifically examining recovery of $\mathrm{QF}$, for the purposes of this study, a patient was deemed to have passed their QF or QF assessment when they were able to successfully complete both the knee extension strength testing and either the single hop or triple hop test at $90 \%$ of that measured for the contralateral limb. This requirement was set a priori. Due to scheduling challenges, an RTS evaluation within 30 weeks of surgery was considered to lie within the 6-month time frame. RTS testing beyond 30 weeks was deemed a delay due to insufficient patient rehabilitation for safe testing.

\section{Statistical Analysis}

Standard descriptive summaries were used to summarize demographics variables. Comparisons of categorical

Table 2. Postoperative Analgesia and Satisfaction Data

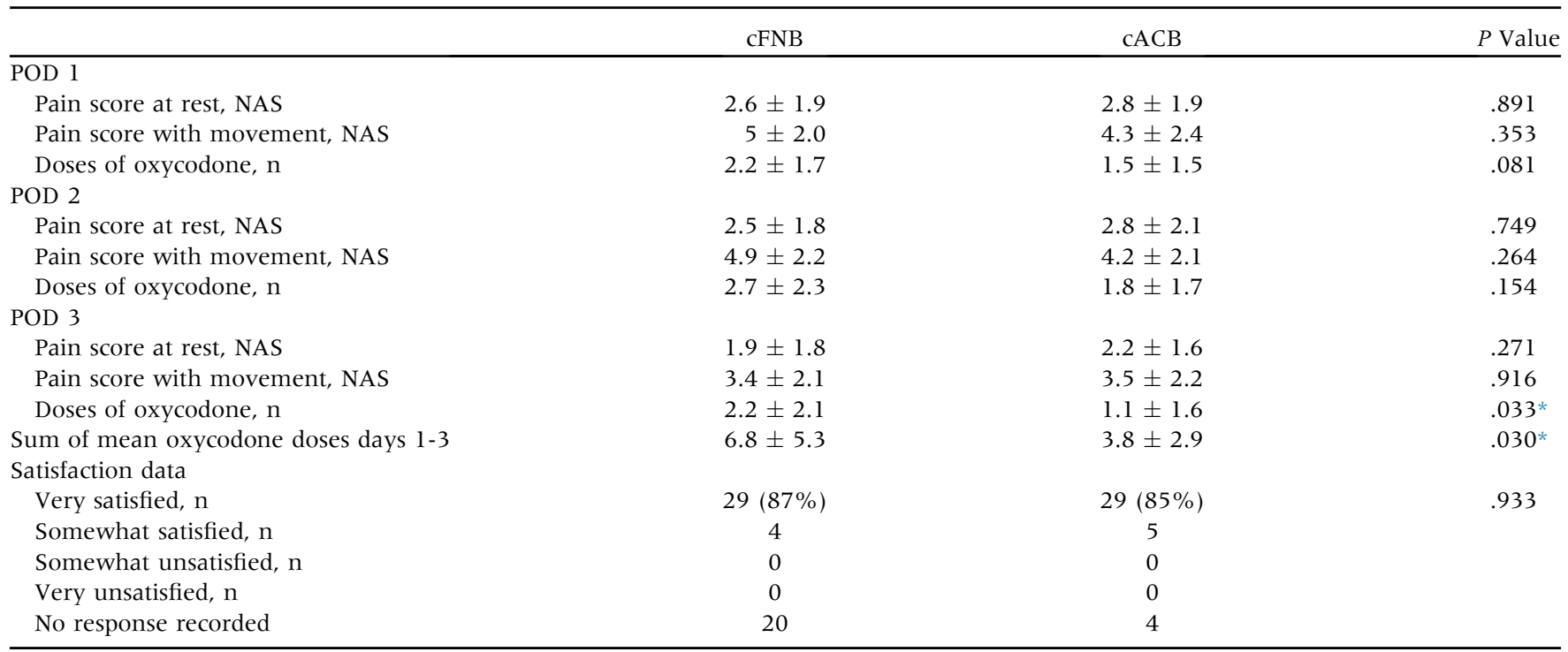

NOTE. Analgesia data are reported as mean \pm standard deviation for pain scores. Satisfaction data reported by number of patients. cACB, adductor canal catheter; cFNB, femoral nerve catheter; NAS, Numerical Analog Scale (0-10); POD, postoperative day.

*Statistically significant. 
Table 3. Complications

\begin{tabular}{lcc}
\hline & cFNB (n=53) & cACB (n = 38$)$ \\
\hline Complications related to cFNB or cACB & $5(9 \%)$ & 0 \\
Removal before POD 3 due to leaking & 2 & 0 \\
Transient bleeding & 1 & 0 \\
Leaking & 0 & 0 \\
Residual numbness in continuous nerve & 2 & 0 \\
$\quad$ block distribution after POD 4 & 0 & 0 \\
Local anesthetic systemic toxicity & 0 & 0 \\
Infection & $7(13 \%)$ & $5(13 \%)$ \\
Complications unrelated to cFNB or cACB & 1 & 1 \\
Residual numbness not in cFNB or cACB distribution & 3 & 1 \\
Needed increase in oxycodone dose & 1 & 0 \\
Urinary retention & 1 & 1 \\
Required admission post-operatively & 1 & 0 \\
Re-injured knee during physical therapy & 0 & 2 \\
Fall after catheter removal, normal sensation & & \\
\hline
\end{tabular}

NOTE. Data reported as number of patients.

CACB, continuous adductor canal block; cFNB, continuous femoral nerve block; POD, postoperative day.

variables between patients with cFNB and those with CACB were made using a $\chi^{2}$ test or Fisher exact test depending on cell size. Comparisons of continuous variables and categorical variables were explored using a Spearman correlation test. A one-way analysis of variance with Bonferroni adjustment was used to compare variations within groups in the daily use of oxycodone PODs 13. Alpha was set at a significance level of $P<.05$. A post hoc power analysis was performed based on the standard deviations in times to return of adequate QF of the 2 groups. All tests were performed using STATA14.2 (StataCorp LLC, College Station, TX).

\section{Results}

In total, 176 patients were identified as having undergone an ACLR during the time interval of interest, 91 of whom met inclusion criteria ( 53 cFNB, 38 cACB) for analysis of early postoperative pain. An additional 3 patients undergoing $\mathrm{CFNB}$ and 5 patients undergoing CACB were excluded for comparisons of late quadriceps strength and function due inadequate PT follow-up. The mean age of patients was 15.1 years (range 11-21 years). Analysis of demographic and surgical data revealed no differences in the make-up of the 2 groups (Table 1). There were no significant differences in the postoperative oxycodone use or pain scores for POD 1POD2. However, at POD 3 patients who received cFNB had statistically significant greater opioid consumption $(2.2 \pm 2.1$ doses cFNB vs $1.1 \pm 1.6$ doses cACB, $P=.033)$. The mean total doses of opioids consumed for days 1 to 3 was greater in the cFNB group (mean 6.8 doses vs 3.8 doses cACB, $P=.003$ ). Comparison of the variation in daily consumption of oxycodone doses between groups revealed no significant differences $(P=.213)$ (Table 2$)$.

There were no major complications related to the peripheral nerve catheters in either group: no local anesthetic toxicity, infections, falls or injuries secondary to muscle weakness or an insensate limb (Table 3). Two patients required unplanned admission after surgery: 1

Table 4. Physical Therapy and Return to Sports Evaluation QFa Data

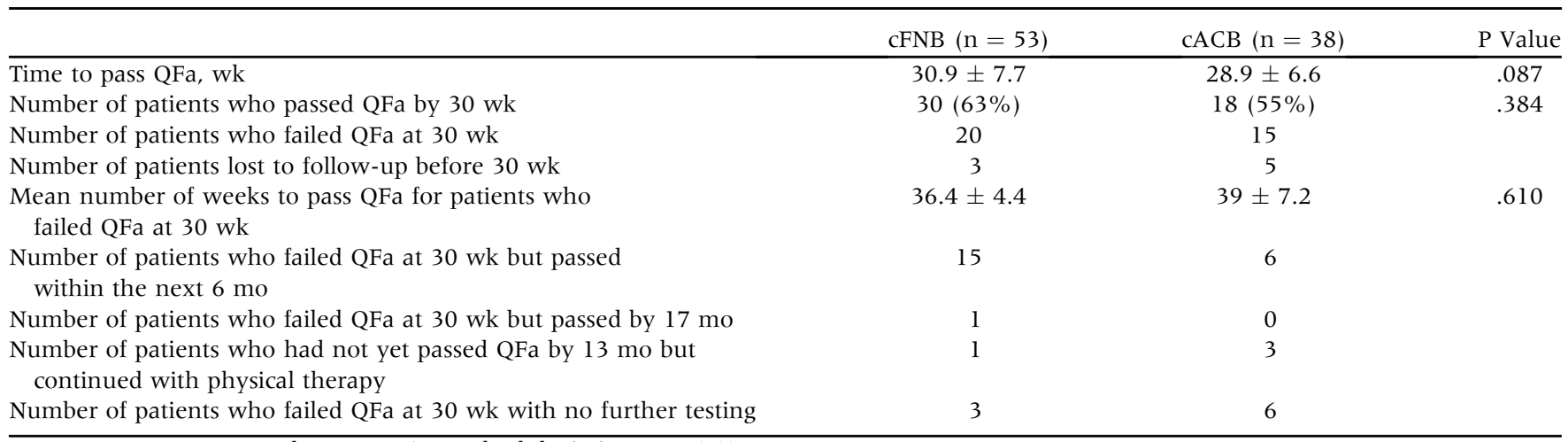

NOTE. Data are reported as mean \pm standard deviation or $\mathrm{n}(\%)$.

CACB, continuous adductor canal block; cFNB, continuous femoral nerve block; QFa, quadriceps function assessment. 
patient undergoing cFNB had muscle spasms requiring intravenous diazepam, whereas 1 patient undergoing CACB had pain requiring intravenous opioids as well as behavior changes suggestive of delirium. Symptoms in each resolved by POD 1. Residual numbness was seen in patients in both groups ( 3 in $\mathrm{CFNB}, 1$ in $\mathrm{CACB}$ ). One patient in each group had prolonged numbness in the distribution of their continuous nerve block, whereas 2 patients in the cFNB had numbness outside of their continuous nerve block distributions. All numbness resolved within 1-year postoperatively. No patients were found to have persistent muscle weakness or long-standing neurologic deficits. Four patients (3 in CFNB, 1 in CACB) needed an increase in their oxycodone dose during PODs 1 to 3. However, all 4 of these had initial doses of oxycodone less than a typical dose of $0.1 \mathrm{mg} / \mathrm{kg}$, due to the stratification of either $5-$ or 7.5 mg doses.

There was no difference in time to passing the QF assessment when we compared the 2 groups (30.9 weeks cFNB, 28.9 weeks CACB, $P=.087$ ) (Table 4). The percent of patients passing the QF assessment at 30 weeks was similar between groups $(30 / 53$ or $63 \%$ cFNB, $18 / 38$ or $55 \%$ cACB, $P=.384$ ). For those who did not pass by 30 weeks, the mean time to passing the QF assessment was also similar in the 2 groups: 36 weeks $\mathrm{CFNB}, 39$ weeks $\mathrm{cACB}$ group $(P=.61)$. The power of the study to detect a 2 -week minimally clinically important difference in time to return of adequate QF between the 2 groups was 0.55 . The post-hoc power analysis determined that we would need a total of 402 patients, 201 in each group, to detect a 2-week difference between the FNC and ANC groups with $80 \%$ power using a 2 -sided $5 \%$ level test.

\section{Discussion}

In this comparison of adolescents undergoing ACLR with a CACB or CFNB, we found few differences in the early postoperative analgesic effectiveness and 6-month postoperative QF. With regard to our hypotheses, we found no differences in analgesic effectiveness of the 2 blocks, as total opioid consumption and overall satisfaction were similar between groups, although satisfaction data were unavailable for 20 of 53 patients in the cFNB group and 4 of 38 patients in the cACB group. There were no differences in the rate of successful QF testing at 6 months nor in the time to successfully completing our QF assessment. Given these findings, we routinely use a CACB over a cFNB for ACLR, as the CACB poses a potentially lower risk of injury to motor nerves to the vastus lateralis, vastus intermedius, and rectus femoris while favoring early weight-bearing.

Our results were similar to the recent literature with regard to early postoperative pain after these blocks. Although we had no measures of early quadriceps functional recovery, a randomized controlled trial comparing $\mathrm{CACB}$ with cFNB after total knee arthroplasty by Jaeger et al., ${ }^{24}$ also found no differences in opioid consumption or early postoperative pain scores but significantly decreased QF in the first 24 hours in the cFNB group. Gao et al. ${ }^{17}$ performed a meta-analysis of 7 randomized controlled trials comparing cACB to cFNB after total knee arthroplasty and found no differences in early postoperative pain scores or opioid use, although early return of quadriceps strength, mobilization, and early hospital discharge favored the cACB group. Patel et al. ${ }^{25}$ performed a randomized control trial of patients undergoing ACLR with a FNB vs $\mathrm{ACB}$ and found no differences in postoperative narcotic requirements in days 1 to 4 .

Although Luo et al. ${ }^{11}$ and Christensen et al. ${ }^{12}$ identified delayed recovery of QF beyond the early postoperative period after FNBs, we found no difference in QF at 6 months and beyond when comparing the cFNB with CACB patients. Luo et al. compared patients having a FNB with an ACLR to a control group with no regional anesthetic, examining isokinetic knee extension and flexion strength, vertical jump, single leg hops, and readiness for return to sports at 6 months. ${ }^{11}$ Although there were greater deficits in fast isokinetic extension strength in the FNB group, there were no differences between groups in vertical jump and single leg hops. ${ }^{11}$ A greater percentage of the control group met criteria for RTS at 6 months $(90 \%$ vs $67 \%$, odds ratio $4.37, P=.002) .{ }^{11}$ Unfortunately, no details on the block anesthetic or whether it was a single-shot or continuous infusion were reported. In a similar comparison study by Krych et al., ${ }^{26}$ a group receiving a continuous 48-hour infusion FNB after ACLR was compared with a control group (no block) for strength, function, and RTS readiness at 7 months. Both strength and functional deficits were noted in the FNB group at 6 months, although there were no significant differences in RTS readiness when comparing the control group (no block) with the FNB group (93\% at $7.3 \mathrm{~m}$ vs $86 \%$ at $7.5 \mathrm{~m}$, respectively). Similar to our results, Runner et al. ${ }^{27}$ examined quadriceps strength and function at 3 and 6 months after single-shot FNB vs ACB and found no differences between groups at either time point.

Although there were no patients in our study who had completely opioid-free PODs, the average number of opioid doses per 24-hour period was less than 3 doses for each POD in each group. Although the mean total opioid consumption PODs 1 to 3 was statistically greater at 6.8 doses cFNB vs 3.8 doses CACB, one may question whether such a difference in total opioid consumption is clinically significant; we would argue that compared with the maximum possible consumption of 18 doses over the first 3 days, both of these totals are quite low, and the difference between 6.8 doses and 3.8 doses may be clinically unimportant. Similar results in 
postoperative pain scores and opioid use in both groups was likely a combination of both the regional technique and an effective multimodal analgesia plan.

\section{Limitations}

There were several limitations in our study. This was a retrospective comparison study, and although there were 2 nearly identical groups of patients examined, there was no randomization into groups, and care for each group was delivered over different periods of time. Incomplete follow-up data on early postoperative pain and opioid use resulted in exclusion of 19 of 112 , or $17 \%$, of potential patients (Fig 1). In addition, 8 of 91 , or $9 \%$, were included for their early postoperative pain results yet were lost to follow-up before RTS testing (Table 4). Lack of early follow-up was due to a multitude of reasons. Family members may have been more likely to answer phone inquiries in the early postoperative period when they had questions, concerns, or complaints, potentially leading to a selection bias. In addition, families often chose or were not available to answer calls from the Regional Anesthesia Service, leading to gaps in our postoperative pain scores and opioid consumption, and hence a reporting bias. Lower mean total opioid consumption PODs 1 to 3 in the cACB group despite greater mean pain scores at rest may speak to a more recent anti-opioid bias among patients and families, although, as previously noted, opioid-prescribing routines were not changed until after this study period.

Time to return of adequate quadriceps strength to walk and jog without a limp was not available from the data available to us, as approximately one half of patients sought routine outpatient physical therapy offsite, where assessments of gait were not routinely reported. This prohibited us from determining the timing of early return of QF and whether it may have varied between groups. Although we routinely scheduled a 6-month RTS assessment for all postoperative patients proceeding without concern through their rehabilitation programs, failure to schedule patients who were ready for testing did occur from time to time. These patients were typically tested for their RTS readiness within 1 month of their 6-month visit. Of those who did have RTS readiness assessments but who either failed the assessment or were unable to finish the assessment due to fatigue or inability to safely perform the requisite elements of the assessments, many (3/53 cFNB, 6/38 cACB) failed to return for a follow-up assessment, thus limiting our ability to determine their time to return of adequate QF.

The post-hoc power analysis revealed that we were underpowered to identify a significant difference between groups for return of QF. However, despite this, we suspect that a 2-week difference at 6 months, if real, is likely only marginally clinically important to most patients and families. In addition, with a recent report suggesting that for every month delay beyond 6 months in return to sport, the ACL re-rupture rate drops by $50 \%$ up to 9 months postsurgery, we are now delaying RTS to no sooner than 9 months. $^{28}$

\section{Conclusions}

We found few differences in postoperative analgesic requirements when comparing patients who underwent ACLR with hamstring autograft with a CACB with those who underwent a similar procedure with a cFNB. Return of quadriceps strength and function by 6 months did not appear to vary with regional technique, either $\mathrm{CACB}$ or $\mathrm{CFNB}$, employed at surgery.

\section{References}

1. Buller LT, Best MJ, Baraga MG, Kaplan LD. Trends in anterior cruciate ligament reconstruction in the United States. Orthop J Sports Med 2015;3.

2. Secrist ES, Freedman KB, Ciccotti MG, Mazur DW, Hammoud S. Pain management after outpatient anterior cruciate ligament reconstruction: A systematic review of randomized controlled trials. Am J Sports Med 2016;44: 2435-2447.

3. Williams BA, Kentor ML, Vogt MT, et al. Economics of nerve block pain management after anterior cruciate ligament reconstruction: Potential hospital cost savings via associated postanesthesia care unit bypass and same-day discharge. Anesthesiology 2004;100:697-706.

4. Hall-Burton DM, Hudson ME, Grudziak JS, Cunningham S, Boretsky K, Boretsky KR. Regional anesthesia is cost-effective in preventing unanticipated hospital admission in pediatric patients having anterior cruciate ligament reconstruction. Reg Anesth Pain Med 2016;41:527-531.

5. Lentz TA, Tillman SM, Indelicato PA, Moser MW, George SZ, Chmielewski TL. Factors associated with function after anterior cruciate ligament reconstruction. Sports Health 2009;1:47-53.

6. Chmielewski TL, Jones D, Day T, Tillman SM, Lentz TA, George SZ. The association of pain and fear of movement/ reinjury with function during anterior cruciate ligament reconstruction rehabilitation. J Orthop Sports Phys Ther 2008;38:746-753.

7. Tyler TF, McHugh MP, Gleim GW, Nicholas SJ. The effect of immediate weightbearing after anterior cruciate ligament reconstruction. Clin Orthop Relat Res 1998;357: $141-148$.

8. Janssen RPA, van Melick N, van Mourik JBA, Reijman M, van Rhijn LW. ACL reconstruction with hamstring tendon autograft and accelerated brace-free rehabilitation: A systematic review of clinical outcomes. BMJ Open Sport Exerc Med 2018;4:e000301.

9. Williams BA, Bottegal MT, Kentor ML, Irrgang JJ, Williams JP. Rebound pain scores as a function of femoral nerve block duration after anterior cruciate ligament reconstruction: Retrospective analysis of a prospective, randomized clinical trial. Reg Anesth Pain Med 2007;32: 186-192. 
10. Ding DY, Manoli A III, Galos DK, Jain S, Tejwani NC. Continuous popliteal sciatic nerve block versus single injection nerve block for ankle fracture surgery: A prospective randomized comparative trial. J Orthop Trauma 2015;29:393-398.

11. Luo TD, Ashraf A, Dahm DL, Stuart MJ, McIntosh AL. Femoral nerve block is associated with persistent strength deficits at 6 months after anterior cruciate ligament reconstruction in pediatric and adolescent patients. Am J Sports Med 2015;43:331-336.

12. Christensen JE, Taylor NE, Hetzel SJ, Shepler JA, Scerpella TA. Isokinetic strength deficit 6 months after adductor canal blockade for anterior cruciate ligament reconstruction. Orthop J Sports Med 2017;5.

13. El Ahl MS. Femoral nerve block versus adductor canal block for postoperative pain control after anterior cruciate ligament reconstruction: A randomized controlled double blind study. Saudi J Anaesth 2015;9:279-282.

14. Charous MT, Madison SJ, Suresh PJ, et al. Continuous femoral nerve blocks: Varying local anesthetic delivery method (bolus versus basal) to minimize quadriceps motor block while maintaining sensory block. Anesthesiology 2011;115:e000301.

15. Ilfeld BM, Duke KB, Donohue MC. The association between lower extremity continuous peripheral nerve blocks and patient falls after knee and hip arthroplasty. Anesth Analg 2010;111:1552-1554.

16. Everhart JS, Hughes L, Abouljoud MM, Swank K, Lewis C, Flanigan DC. Femoral nerve block at time of ACL reconstruction causes lasting quadriceps strength deficits and may increase short-term risk of re-injury [published online July 17, 2019]. Knee Surg Sports Traumatol Arthrosc. https://doi.org/10.1007/s00167-019-05628-7.

17. Gao F, Ma J, Sun W, Guo W, Li Z, Wang W. Adductor canal block versus femoral nerve block for analgesia after total knee arthroplasty: A systematic review and metaanalysis. Clin J Pain 2017;33:356-368.

18. Lund J, Jenstrup MT, Jaeger P, Sørensen AM, Dahl JB. Continuous adductor-canal-blockade for adjuvant postoperative analgesia after major knee surgery: Preliminary results. Acta Anaesthesiol Scand 2011;55:14-19.
19. Jaeger P, Nielsen ZJ, Henningsen MH, Histed KL, Mathiesen O, Dahl JB. Adductor canal block versus femoral canal block and quadriceps strength. Anesthesiology 2013;118:409-415.

20. Saba R, Brovman EY, Kang D, Greenberg P, Kaye AD, Urman RD. A Contemporary Medicolegal analysis of injury related to peripheral nerve blocks. Pain Physician 2019;22:389-400.

21. Barber-Westin SD, Noyes FR. Factors used to determine return to unrestricted sports activities after anterior cruciate ligament reconstruction. Arthroscopy 2011;27: 1697-1705.

22. Thomee R, Kaplan Y, Kvist J, et al. Muscle strength and hop performance criteria prior to return to sports after ACL reconstruction. Knee Surg Sports Traumatol Arthrosc $2011 ; 19: 1798-1805$.

23. Hewett TE, Di Stasi SL, Myer GD. Current concepts for injury prevention in athletes after anterior cruciate ligament reconstruction. Am J Sports Med 2013;41: 216-224.

24. Jaeger P, Zaric D, Fomsgaard JS, et al. Adductor canal block versus femoral nerve block for analgesia after total knee arthroplasty: A randomized, double-blind study. Reg Anesth Pain Med 2013;38:526-532.

25. Patel R, Lynch J, Okoroha KR, Lizzio V, Meta F, Moutzouros V. Adductor canal block versus femoral nerve block for pain control after anterior cruciate ligament reconstruction: A prospective randomized trial. Arthroscopy 2018;34:e35.

26. Krych A, Arutyunyan G, Kuzma S, Levy B, Dahm D, Stuart M. Adverse effect of femoral nerve blockade on quadriceps strength and function after ACL reconstruction. J Knee Surg 2015;28:83-88.

27. Runner RP, Boden SA, Godfrey WS, et al. Quadriceps strength deficits after femoral nerve block versus adductor canal block for anterior cruciate ligament reconstruction. Orthop J Sports Med 2018;6.

28. Grindem H, Snyder-Mackler L, Moksnes H, Engebretsen L, Risberg MA. Simple decision rules can reduce reinjury risk by $84 \%$ after ACL reconstruction: The Delaware-Oslo ACL cohort study. Br J Sports Med 2016;50:804-829. 